\title{
RIEMANNIAN INTEGRAL OF SET-VALUED FUNCTION
}

\author{
E.S.Polovinkin \\ Moscow State University \\ Moscow, USSR
}

Lebesgue integral of a set-valued function is a conception of growing importance for the theories of the optimal control and of the differential games (see for example [1]). For solving concrete problems it will be often useful to reduce a Lebegue setintegral to a riemannien one. In this article we shall find sufficient and necessary conditions of existence of riemannien integral of a set-valued function and prove the equality between riemannien and Lebesgue set-integrals in the case when riemannien set-integral exists.

I. Notations. Definitions. Auxiliary Propositions.

Let $R^{n}$ be an euclidean n-space, $(x, y)$ - the scalar product of $x, y \in R^{n}, I=[a, b] \subset R^{1}, V=\left\{x \in R^{n}\|\| x \| \leqslant 1\right\}, W=\left\{\psi \in R^{n}\|\| \psi \|=1\right\}$. Let $A$ and $B$ be compact subsets of $R^{n}$. The Hausd orffistance is definded by formula: $h(A, B)=\min \{z \geqslant 0 \mid A \subset B+z V, B C A+r V\}$. We shall denote by $\Omega^{n}$ the space of all compact subsets of $R^{n}$ with Hausdorff metric in it. If $\left\{A_{i}\right\} \in \Omega^{n}$, then we shall define $\sum_{i=1}^{m} A_{i}=A_{1}+\ldots+A_{m}=\left\{a_{1}+\ldots\right.$ $\left.\ldots+a_{m} \mid a_{i} \in A_{i}\right\}$; if $A \in \Omega^{n}$ - then tend $A^{\prime=1}$ is closure of extreme points of $A$ and $|A|=h(A, 0)$.

We shall say that $F: I \rightarrow \Omega^{n}$ is set-valued function. The conceptions of limit in the metric space $\Omega^{n}$ (denote $L / M$ ) and of continuity of function $F: I \rightarrow \Omega^{n}$ are defined as usual. We shall say that map $F: I \rightarrow \Omega^{n}$ is convex-valued function if all the sets $F(t) C R^{n}$ are convex for every fixed $t \in I$. Support function of the set $A C R^{n}$ is the function $s(\psi, A)=\sup \{(\psi, x) \mid x \in A\}$. We shall consider the support function only for $\Psi \in W$.

We shall say that map $F: I \rightarrow \Omega^{n}$ is Lebesgue measurable function on $I$ if for any closed set $D \subset R^{n}$ the set $\{t \in I \mid F(t) \cap D \neq \phi\}$ is Lebesgue measurable set.

Definition 1 Lebesgue integral of the set-valued function $F: I \rightarrow \Omega^{n}$ on $I$ (set-integral) is the set $L \int_{T} F(t) d t=\left\{w \in R^{n} / \exists r(t) \in F(t)\right.$; $r(t)$ is Lebesgue measurable function on $I$ and such that $\left.w=L \int_{I} r(t) d t\right\}$.

Subdivision of an interval $I=[a, B]$ is finite set of numbers $\omega=\left\{t_{1}, t_{2}, \ldots, t_{N}\right\}$ such that $t_{i}=a, t_{i}<t_{i+1}, t_{N}=\boldsymbol{b}$. Let $\Delta t_{i}=t_{i+1}-t_{i}$, $\lambda=\max \left\{\Delta t_{i}\right\}$ be diameter of the subdivision $\omega$. Let $\xi_{i} \in\left[t_{i}, t_{i+1}\right]$ $1 \leq i \leq N-1$ 
be an arbitrary point. We shall consider in this article only bounded maps $F: I \rightarrow \Omega^{n}$, that means: $\exists \alpha>0:|F(t)|<\alpha \forall t \in I$.

Definition 2 Riemannien integral of the bounded set-valued

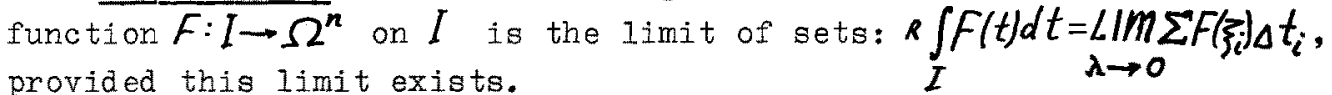
We shall use following well-known propositions:

Proposition 1. Convex-valued function $F: I \rightarrow \Omega^{n}$ is continuous iff it is bounded and support function $s(\Psi, F(t))$ is continuous by $t$ for any $\psi \in W .([2])$.

Proposition 2. (C-property). Map $F: I \rightarrow \Omega^{n}$ is messurable on $I$ iff for any $\varepsilon>0$ there exists closed set $I_{1} \subset I$ such that Lebesgue measure $m\left(I \backslash I_{1}\right)<\varepsilon$ and $F(t)$ is continuous on $I_{1}([3])$.

Proposition 3. Let $F: I \rightarrow \Omega^{n}$ be measurable function on $I$ and let there exist the Lebesgue summable function $\mu(t)>0$ on $I$ such that $|F(t)| \leqslant \mu(t)$. Then the Lebesgue integral of $F(t)$ is non empty convex compact subset of $R^{n}$ and $L \int_{I} F(t) d t=L \int_{I} \operatorname{co} F(t) d t$.

\section{The main lemmas.}

Lemma 1. The bounded convex-valued function $F: I \rightarrow \Omega^{n}$ is continuous a.e. (almost everywhere) on $I$ iff the function $f(t)=$ $=\boldsymbol{S}(\boldsymbol{\Psi}, \boldsymbol{F}(t))$ is continuous a.e. on $I$ for any $\boldsymbol{\Psi} \in \boldsymbol{W}$.

The proof follows from proposition 1.

Lemma 2. Let the suppositions of proposition 3 be satisfied. Then $s\left(\Psi, \iota \int_{I} F(t) d t\right)=L \int_{I} s(\Psi, F(t)) d t$.

Proof. By proposition 3 the integral $\angle \int_{I} F(t) d t$ is convex compact, therefore for any $\psi \in W$ there exists vector $x \in L \int_{I} F(t) d t$ such that $S\left(\Psi, L \int_{I} F(t) d t\right)=(\Psi, X)$. Then by definition 1 there exists measurable function $r(t) \in F(t)$ such that $x=L \int_{r} r(t) d t$. Hence $s\left(\Psi, \angle \int_{I} F(t) d t\right)=\left(\psi, \angle \int_{I} r(t) d t\right)=\left\langle\int_{I}(\psi, r(t)) d t \leqslant \angle \int_{I} s(\psi, F(t)) d t\right.$. On the other hand Iet us consider map $R(t, \psi)=\{x \in F(t) \mid s(\psi, F(t))=$ $=(\psi, x)\}$. This map is measurable because $R(t, \psi)=F(t) \cap Q(t, \psi)$ where $Q(t, \psi)=\left\{x \in R^{n} \mid s(\psi, F(t))=(\psi, x)\right\}$. But the map $Q(t, \psi)$ is measurable because $h\left(Q(t, \psi), Q\left(t^{*}, \psi\right)\right)=\left|s(\psi, F(t))-s\left(\psi, F\left(t^{*}\right)\right)\right|$. But if $R(t, \psi)$ is measurable then there exists the measurable function $r(t) \in R(t, \psi)$. Hence we have $s(\psi, F(t))=(\psi, r(t))$. Finally

$$
L \int_{I} s(\psi, F(t)) d t=L \int_{I}(\psi, r(t)) d t=\left(\psi, L \int_{I} \mu(t) d t\right) \leqslant s\left(\psi, L \int_{I} F(t) d t\right) .
$$


Iemma 3. If convex-valued function $F: I \rightarrow \Omega^{n}$ is continuous a.e. on $I$ then function tend $F: I \rightarrow \Omega^{n}$ is continuous a.e. on $I$. Proof. Let $W\left(\psi_{1}, \ldots, \psi_{k}\right)=\left\{\psi \in W /\left(\psi, \psi_{i}\right)=0 ; 1 \leqslant i \leqslant \kappa\right\}$. Let us consider a set of mutually orthogonal points $\psi_{1}, \ldots, \psi_{n}$ from $W$. We shall define the maps $R\left(t, \psi_{1}, \ldots, \psi_{k}\right)$ in such a way:

$$
\begin{aligned}
& R\left(t, \Psi_{1}\right)=\left\{x \in F(t) \mid s\left(\Psi_{1}, F(t)\right)=\left(\Psi_{1}, x\right)\right\} \text {, } \\
& R\left(t, \Psi_{1}, \Psi_{2}\right)=\left\{x \in R\left(t, \Psi_{1}\right) \mid s\left(\Psi_{2}, R\left(t, \Psi_{1}\right)\right)=\left(\Psi_{2}, x\right)\right\} \text {, } \\
& R\left(t, \Psi_{1}, \ldots, \Psi_{n}\right)=\left\{x \in R\left(t, \Psi_{1}, \ldots, \Psi_{n-1}\right) / s\left(\Psi_{n}, R\left(t, \Psi_{1}, \ldots, \psi_{n-1}\right)\right)=\left(\Psi_{n}, x\right)\right\} .
\end{aligned}
$$

It is easy to show (as in lemma 2) that function $t \rightarrow R\left(t, \psi_{1}, \ldots, \Psi_{n}\right.$ ) ) is continuous a.e. on $I$ and that tend $F(t)=$

$$
=d \bigcup_{\psi_{1} \in W} \bigcup_{\psi_{2} \in W\left(\psi_{1}\right)} \cdots \bigcup_{\psi_{n} \in W\left(\psi_{1}, \ldots, \psi_{n-1}\right)} R\left(t, \psi_{1}, \ldots, \psi_{n}\right) .
$$

Lemme 4. Let function $F: I \rightarrow \Omega^{n}$ be summable on $I$ by Riemann (that is there exists riemannien integral of $F(t)$ ). Then for any $\psi \in W$ the support function $f(t)=s(\psi, F(t))$ is summable by Riemann and $s\left(\psi, R \int_{I} F(t) d t\right)=R \int_{I} S(\psi, F(t)) d t$.

Proof. It is easy to show that if $\operatorname{LIm}_{n \rightarrow \infty} P_{h}=P$, then for any $\psi \in W$ $S(\psi, P)=\lim _{n \rightarrow \infty} S\left(\Psi, P_{n}\right)$. From here follows

$$
\begin{aligned}
& s\left(\psi, R \int_{I} F(t) d t\right)=s\left(\psi, \lim _{\lambda \rightarrow 0} \sum F\left(\xi_{i}\right) \Delta t_{i}\right)=\lim _{\lambda \rightarrow 0} s\left(\psi, \Sigma F\left(\xi_{i}\right) \Delta t_{i}\right)= \\
& =\lim _{\lambda \rightarrow 0} \sum s\left(\psi, F\left(\xi_{i}\right)\right) \Delta t_{i}=R \int_{I} s(\psi, F(t)) d t .
\end{aligned}
$$

Lemma 5. Let $\left\{F_{\lambda}\right\} \in \Omega^{n}, \lambda>0, F \in \Omega^{n},\left|F_{\lambda}\right| \leqslant \alpha$. Let sets $F_{\lambda}$ and $F$ be convex and for any $\psi \in W \lim _{\lambda \rightarrow 0} s\left(\psi, F_{\lambda}\right)=s(\psi, F)$. Then there exists the limit of sets $F_{\lambda}$ in space $\Omega^{n}$ and $\lim _{\lambda \rightarrow 0} F_{\lambda}=F$. $\lambda \rightarrow 0$

Proof of this lemma is similar to the proof of proposition 1. Lemme 6. Let $F: I \rightarrow \Omega^{n}$ be convex-valued function on $I$ and for any $\psi \in W$ the support function $f(t)=s(\psi, F(t))$ be summable by Riemann on $I$. Then $F(t)$ is summeble by Riemenn and $R \int_{I} F(t) d t=L \int_{I} F(t) d t$.

Proof. By lemma 2 we have $s\left(\psi, L \int_{I} F(t) d t\right)=L \int_{I} s(\psi, F(t)) d t=$ $=R \int_{I} s(\psi, F(t)) d t=\lim _{\lambda \rightarrow 0} \sum s\left(\psi, F\left(\xi_{i}\right)\right)_{\Delta} t_{i}=\lim _{\lambda \rightarrow 0} s\left(\psi, \Sigma F\left(\xi_{i}\right) \Delta t_{i}\right)$. 
From here and lemma 5 follows the proof.

Lemma 7. Let $P \in \Omega^{n}$. Then $R \int_{I} P d t=(b-a) \operatorname{co} P$.

Proof. Let $\omega=\left\{t_{1}, \ldots, t_{N}\right\}$ be subdivision of $I=[a, b]$. Consider sets $P_{A}=\sum_{i=1}^{N-1}\left(P \cdot \Delta t_{i}\right)$. It is easy to show that $P_{A} \subset(b-a) \operatorname{co} P$. on the other hand let $x \in(b-a) c o P$. By carateodory's theorem there exist some points $x_{i} \in \operatorname{coP}$ and numbers $M_{i}>0, i=1, \ldots, k ; \kappa \leqslant n+1$, $\sum_{i=1}^{K} \mu_{i}=1$, such that $x=(b-a)\left(\mu_{1} x_{1}+\ldots+\mu_{k} x_{k}\right)$. Let $|P| \leqslant \alpha$. Denote $\mu(x)=(b-a) \min \left\{\mu_{i} \mid 1 \leqslant i \leqslant k\right\}$ and $\lambda(x)=\min \left\{\mu(x), \frac{\varepsilon}{2 n \alpha}\right\}$.

It is obvious that $\lambda(x)>0$ and $x \in P_{\lambda}+\frac{\varepsilon}{2} V$ for any subdivision with diameter $\lambda \leqslant \lambda(x)$. If $B_{\varepsilon}(x)=\left\{y \in R^{n} \mid\|x-y\|<\frac{\varepsilon}{2}\right\}$ then $B_{\varepsilon}(x) \subset P_{\lambda}+\varepsilon \cdot V$. If we take a finjte covering of compact set $(b-a) \operatorname{co} P$ by neighborhoods $B_{\varepsilon}(x)$ then we shall find $\lambda_{0}>0$ such that for any subdivision with $\lambda \leqslant \lambda_{0}:(b-a) \operatorname{co} P \subset P_{\lambda}+\varepsilon V$. Finally $h\left(P_{\lambda},(b-a) \operatorname{co} P\right)<\varepsilon$.

\section{The main theorems.}

Theorem 1 . Let $F: I \rightarrow \Omega^{n}$ be a convex-valued function on $I$. Map $F: I \rightarrow \Omega^{n}$ is summable by Riemann on $I$ iff $F: I \rightarrow \Omega^{n}$ is continuous a.e. on $I$. With such conditions riemannien integral of $F$ is equal to Lebesgue integral of $F$.

Proof. It is well-known that a usual function is summable by Riemann on $I$ iff it is continuous $a . e$. on $I$. Therefore the proof is consequence of lemmas 4,1 and 6 .

Theorem 2. If $F: I \rightarrow \Omega^{n}$ is continuous function on $I$ then it is summable by Riemann and $R \int_{I} F(t) d t=R \int_{I} \operatorname{co} F(t) d t$.

Proof. By theorem 1 and by continuity of $F(t)$ for any $\varepsilon>0$ there exists $\delta>0$ such that for $t, t_{*} \in I,\left|t-t_{*}\right|<\delta$, and for any subdivision $\omega_{1}=\left\{\tau_{1}, \ldots, \tau_{N}\right\}$ with diameter $\delta$ we have:

$F(t) \subset F\left(t_{*}\right)+\frac{\varepsilon}{3(b-a)} V ; h\left(\sum_{i=1}^{N-1} \operatorname{co} F\left(\gamma_{i}\right) \Delta \tau_{i}, R \int_{I} \operatorname{co} F(t) d t\right)<\frac{\varepsilon}{3}$, where $\gamma_{i} \in\left[\tau_{i}, \tau_{i+1}\right], \Delta \tau_{i}=\tau_{i+1}-\tau_{i}$

Let us consider subdivision $\omega_{2}=\left\{t_{11}, t_{12}, \ldots, t_{1 k_{1}}=t_{21}\right.$, $\left.t_{22}, \ldots, t_{2 k_{2}}=t_{31}, \ldots, t_{N 1}\right\}$ 
with $t_{11}=\tau_{1}=a ; t_{21}=\tau_{2}, \ldots, t_{N 1}=\tau_{N}=b$, and let diameter $\omega_{2}$ be $\boldsymbol{\lambda}$. The integral sum for $\boldsymbol{\omega}_{2}$ is of a form:

$\sum_{i=1}^{N-1} \sum_{j=1}^{K_{i}-1} F\left(\xi_{i j}\right) \Delta t_{i j} \quad$ where $\xi_{i j} \in\left[t_{i j}, t_{i j+1}\right], \Delta t_{i j}=t_{i j+1}-t_{i j}$. On every segment $\left[\tau_{i}, \tau_{i+1}\right] \quad$ while $\lambda_{\tau_{i+1} \rightarrow 0}$ the sum $\sum_{j=1}^{k_{i-1}} F\left(\gamma_{i}\right) \Delta t_{i j}$ tend $s$ to limit which is equal to $R \int_{\tau_{i}}^{\tau_{i+1}} F\left(\gamma_{i}\right) d t=\operatorname{co} F\left(\gamma_{i}\right) \Delta \tau_{i}$. Let us choose $\lambda_{0}>0$ such that for any $\omega_{2}$ with diameter $\lambda \leq \lambda_{0}$ for every $i=1, \ldots, N-1$ we get: $h\left(\sum_{j=1}^{K_{i}-1} F\left(\gamma_{i}\right) \Delta t_{i j}, \operatorname{co} F\left(\gamma_{i}\right) \Delta \tau_{i}\right)<\frac{\mathcal{E}}{3(N-1)}$. From here $h\left(\sum_{i=1}^{N-1} \sum_{j=1}^{K_{i}-1} F\left(\gamma_{i}\right) \Delta t_{i j}, \sum_{i=1}^{N-1} \operatorname{coF}\left(\gamma_{i}\right) \Delta \tau_{i}\right)<\frac{\varepsilon}{3}$. From $\left|\gamma_{i}-\xi_{i j}\right|<\delta$ : $h\left(\sum_{i=1}^{N-1} \sum_{j=1}^{K_{i}-1} F\left(\xi_{i j}\right) \Delta t_{i j}, \sum_{i=1}^{N-1} \sum_{j=1}^{K_{i}-1} F\left(\gamma_{i}\right) \Delta t_{i j}\right) \leqslant \sum_{i=1}^{N-1} \sum_{j=1}^{K_{i}-1} h\left(F\left(\xi_{i j}\right), F\left(\gamma_{i}\right)\right) \Delta t_{i j}<\frac{\varepsilon}{3}$. Hence for any $\lambda \leqslant \lambda_{0}: h\left(\sum_{i=1}^{N-1} \sum_{j=1}^{K_{i}-1} F\left(\xi_{i j}\right) \Delta t_{i j}, r \int \operatorname{co} F(t) d t\right)<\varepsilon$.

Theorem 3. If map $F: I \rightarrow \Omega^{n}$ is continuous a.e. on $I$ then it is summable by Riemann and $R \int_{I} F(t) d t=R \int_{I} c o F(t) d t$. Proof. Let $I_{I}$ be set of points of cease of ${ }^{I} F(t)$ on $I$, then $m I_{1}=0$. Let $\alpha>0$ be such that $|F(t)| \leqslant \alpha$ for $t \in I$. Let $\varepsilon>0$ and $\delta<\frac{\varepsilon}{6 \alpha}$. There exists an open set $I_{2}$ such that $m I_{2}=\delta$ and $I_{1} \subset I_{2} \subset I$. For any subdivision $\omega$ the integral sum may be decomposed into two parts: $\quad \Sigma F\left(\xi_{i}\right) \Delta t_{i}=\Sigma_{I} F\left(\xi_{i}\right) \Delta t_{i}+\Sigma_{\underline{I}} F\left(\xi_{i}\right) \Delta t_{i}$, where $\Sigma_{\text {II }}$ consists of those indexes $i$ for which $\left[t_{i}, t_{i+1}\right] \cap I_{2} \neq \varnothing$. By theorem 2 we can choose $\lambda_{0}>0$ such that for any subdivision with diameter $\lambda \leqslant \lambda_{0}$ we'll have $\sum_{\underline{I}} \Delta t_{i}<2 \delta$ and $h\left(\Sigma_{I} F\left(\xi_{i}\right) \Delta t_{i}, R \int \operatorname{co} F(t) d t\right)<\frac{\varepsilon}{2}$. $\mid h\left(R \int_{I} \operatorname{coF}(t) d t, \Sigma F\left(\xi_{i}\right) \Delta t_{i}\right) \leqslant h\left(R \int_{I I_{2}} \operatorname{coF}(t) d t, \Sigma_{I} F\left(\xi_{i}\right)_{\Delta t_{i}}^{I I I_{2}}\right)+$ Therefore $+h\left(R \int_{I_{2}} \operatorname{co} F(t) d t, \Sigma_{\underline{I}} F\left(\xi_{i}\right) \Delta t_{i}\right)^{I I_{2}}<\frac{\varepsilon}{2}+3 \alpha \delta<\varepsilon$.

Theorem 4. The function $F: I \rightarrow \Omega^{n}$ is summable by Riemann iff the function $c O F: I \rightarrow \Omega^{n}$ is continuous a.e. on $I$. Under this condition the following equalities hold:

$$
R \int_{I} \operatorname{co} F(t) d t=R \int_{I} F(t) d t=R \int_{I} \operatorname{tend} \operatorname{co} F(t) d t ; R \int_{I} F(t) d t=L \int_{I} F(t) d t .
$$
Proof. Let $\operatorname{co} F(t)$ be continuous a.e. on $I$ then tendco $F(t)$ is continuous a.e. on $I$ ( lemma 3). By theorem 3 and by inclusion: $\operatorname{co} F(t) \supset F(t) \supset$ tend $\operatorname{co} F(t)$ we get the existence of riemannien 
integral of $F(t)$ and equality of three integrals. On the other hand if $F(t)$ is summable by Riemann then it is easy to show that $\operatorname{co} F(t)$ is continuous a.e. on $I$ (by lemmas 4 and 1 ). By equality of three integrals, by theorem 1 and proposition 3 we get:

\section{$R \int_{I} F(t) d t=l \int_{I} F(t) d t$.}

\section{REFERENCES}

1. Pontrjagin, I.S., Linear Differential Games. I., D.A.N. SSSR 174. No 6, 1967.

2. Blagodatskikh, V.I., Convexity of Spheres of Achievability Diff. Equations, 8 No 12, 1972, USSR.

3. Plis: A., Remark on Measurable Set-valued Functions. Bull. Acad. Polon. Sci., Sir. Sci. Math. Astr. Phys., 9, 1961.

4. Hermes, $H .$, The Generalized Differential Equation $\dot{x} \in R(t, x)$, Advances in Math., 4, 1970. 